\title{
Spatial dependence of magnetopause energy transfer: Cluster measurements verifying global simulations
}

\author{
M. Palmroth ${ }^{1}$, T. V. Laitinen ${ }^{1}$, C. R. Anekallu, ${ }^{1,}$, T. I. Pulkkinen ${ }^{2}$, M. Dunlop ${ }^{3}$, E. A. Lucek ${ }^{4}$, and I. Dandouras ${ }^{5}$ \\ ${ }^{1}$ Finnish Meteorological Institute, Helsinki, Finland \\ ${ }^{2}$ Aalto University, School of Electrical engineering, Espoo, Finland \\ ${ }^{3}$ Rutherford Appleton Laboratory, Chilton, Didcot, UK \\ ${ }^{4}$ Imperial College, London, UK \\ ${ }^{5}$ CESR, Université de Toulouse, Toulouse, France \\ *also at: University of Helsinki, Department of Physics, Helsinki, Finland
}

Received: 22 November 2010 - Revised: 15 March 2011 - Accepted: 9 May 2011 - Published: 13 May 2011

\begin{abstract}
We investigate the spatial variation of magnetopause energy conversion and transfer using Cluster spacecraft observations of two magnetopause crossing events as well as using a global magnetohydrodynamic (MHD) simulation GUMICS-4. These two events, (16 January 2001, and 26 January 2001) are similar in all other aspects except for the sign of the interplanetary magnetic field (IMF) $\mathrm{y}$-component that has earlier been found to control the spatial dependence of energy transfer. In simulations of the two events using observed solar wind parameters as input, we find that the GUMICS-4 energy transfer agrees with the Cluster observations spatially and is about $30 \%$ lower in magnitude. According to the simulation, most of the the energy transfer takes place in the plane of the IMF (as previous modelling results have suggested), and the locations of the load and generator regions on the magnetopause are controlled by the IMF orientation. Assuming that the model results are as well in accordance with the in situ observations also on other parts of the magnetopause, we are able to pin down the total energy transfer during the two Cluster magnetopause crossings. Here, we estimate that the instantaneous total power transferring through the magnetopause during the two events is at least $1500-2000 \mathrm{GW}$, agreeing with $\epsilon$ scaled using the mean magnetopause area in the simulation. Hence the combination of the simulation results and the Cluster observations indicate that the $\epsilon$ parameter is probably underestimated by a factor of $2-3$.
\end{abstract}

Keywords. Magnetospheric physics (Magnetopause, cusp, and boundary layers; Solar wind-magnetosphere interactions) - Space plasma physics (Numerical simulation studies)

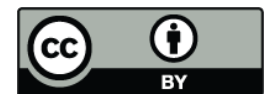

Correspondence to: M. Palmroth (minna.palmroth@fmi.fi)

\section{Introduction}

Dynamical phenomena within the near-Earth space are powered by the solar wind energy. The central large-scale manifestation of the solar wind energy transfer is related to the plasma and magnetic field circulation within the magnetosphere and ionosphere, which is often referred to as "global convection". Dungey (1961) explained global convection as a consequence of magnetic reconnection, where the dayside magnetospheric magnetic field is broken and re-joined with the interplanetary magnetic field (IMF), advected with the solar wind flow towards the magnetospheric tail, where again the oppositely directed open magnetic flux from both hemispheres reconnect and form closed flux tubes. On the other hand, Axford and Hines (1961) related the global convection to viscous interactions on the magnetopause surface. Both mechanisms produce circulation of high-latitude magnetic field and plasma from dayside to nightside and subsequently from nightside to dayside on lower latitudes. The global convection pattern maps into the ionosphere, where a global electric potential pattern forms; in Dungey's model because the interplanetary electric field maps along equipotential field lines directly to the ionosphere, and in the viscous model because the plasma motion within the magnetic field yields also an electric field. While both mechanisms are at work, the fact that the ionospheric potential is very low during times of small dayside reconnection rate (e.g., Boyle et al., 1997) suggests that dayside reconnection is the most important contributor to the solar wind energy transfer.

The current theory for extracting the solar wind power is associated with a load-generator mechanism (Siscoe and Cummings, 1969; Lundin and Evans, 1985) allowed by dayside reconnection. In the dayside reconnection region, magnetic energy is converted into kinetic energy of the plasma as reconnection accelerates plasma away from the reconnection

Published by Copernicus Publications on behalf of the European Geosciences Union. 
(a) IMF clock angle $140^{\circ}$
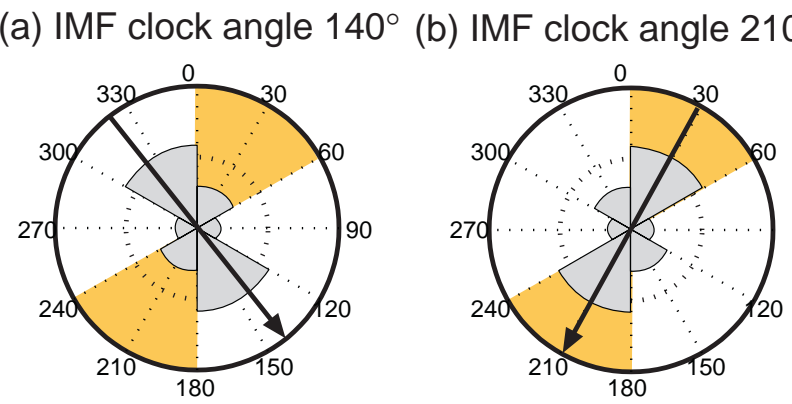

Fig. 1. Event selection strategy. The gray areas show the integrated amount of energy transfer on the magnetopause surface in six azimuthal sectors during IMF clock angle of (a) $\theta=140^{\circ}$ and (b) $\theta=210^{\circ}$ looking tailwards, and the IMF direction is illustrated with a black arrow. The yellow areas in the diagram illustrate the desired areas of Cluster crossings; in panel (a) Cluster would not observe significant energy transfer while in panel (b) the energy transfer would be increased and the amount and sign would depend on the upstream parameters as well as the exact location of crossing. The energy transfer results are from a previous unpublished run and are here only to facilitate an a priori hypothesis for the investigation.

site. After a field line has been reconnected, it evolves across the magnetopause and is added to the tail lobes of open magnetic flux in the nightside, where it eventually reconnects and closed flux is created. Therefore, current theory suggests that on the dayside equatorward of the cusp, energy is transferred to the plasma by magnetic reconnection, which represents a load in the system. On the other hand, tailward of the cusp energy is extracted from the motion of the magnetosheath plasma and converted to magnetic energy, making hence the tail magnetopause a generator. While the qualitative picture of the cause and effect of the energy transfer is clear, the quantitative formulation has proven markedly difficult. Mostly, the global energy transfer estimates rely on correlations of the solar wind parameters to magnetospheric activity indices (Akasofu, 1981; Newell et al., 2007). However, such proxies of the energy transfer lack spatial information of the process and the magnitude of the transferred energy is approximated from the magnetospheric response.

Using a global MHD simulation GUMICS-4, Palmroth et al. $(2003,2006)$ found a general temporal correspondence to the energy transfer proxies, but also found a distinct spatial variation in the energy transfer, where the energy transfers in a plane of the IMF orientation. That is, if the IMF clock angle $\theta=\tan ^{-1}$ (IMF y/IMF $\mathrm{z}$ ) is $180^{\circ}$ and the IMF is purely southward, the energy transfers in the north-south direction on the magnetopause, while deviations from the due south orientation shifts the energy transfer spatial distribution. This was explained by Poynting flux focussing (Papadopoulos et al., 1999; Palmroth et al., 2003), where the electromagnetic energy focusses towards the magnetopause in the plane of the IMF and deviates away from the mag- netopause in a plane perpendicular to the IMF orientation. Mathematically, the Poynting flux focussing is complementary to the load-generator mechanism (Palmroth et al., 2010) and it is enabled because the Poynting vector at the magnetopause surface is nonzero in areas where the open field lines advect tailwards. While the spatial variation of the energy transfer is a trivial consequence of the Poynting theorem, it has never been observationally verified on the magnetopause surface.

An important step towards quantitative energy transfer estimates were taken by Rosenqvist et al. (2006, 2008b), who presented a method to compute energy conversion within the magnetopause current layer using Cluster observations. Later, they compared the Cluster results with ones obtained from a global MHD simulation (Rosenqvist et al., 2008a). In this paper we carry on with their methodology to investigate the spatial energy transfer distribution on the magnetopause but compare the results to another global MHD simulation. Our strategy is illustrated in Fig. 1: Based on earlier global MHD simulation results, the energy transfer occurs in the plane of the IMF such that for example during IMF clock angle is $\theta=140^{\circ}\left(210^{\circ}\right)$, the energy transfers in the northern dawn and the southern dusk (northern dusk and southern dawn) portions of the magnetopause, predominantly sunward of $x=-10 R_{\mathrm{E}}$ (Palmroth et al., 2003, 2006). We search for event pairs in which the upstream parameters are otherwise the same and steady, but for which the IMF y-component is equal but of different sign. The steady upstream conditions are desired as the pressure variations affect the local energy transfer values, while the different sign in IMF y shifts the energy transfer pattern on the magnetopause as illustrated in Fig. 1. From the event pairs, we take only events where the Cluster constellation crosses the magnetopause within the same area, and for which the separation is preferably such that it allows the determination of the current density using the accurate curlometer technique (Dunlop et al., 2002). We expect that for an event similar to that in Fig. 1a, Cluster would not observe much energy conversion, while in an event depicted in Fig. 1b significant energy conversion would be observed.

With the above search strategy, we identified two events; 16 January 2001, and 26 January 2001, for which we carry out the simulations and investigate the energy transfer results. By coincidence, the 26 January 2001 event has been extensively studied, and it includes several magnetopause crossings (Dunlop et al., 2002; Bosqued et al., 2001) for which the energy conversion estimates are obtained (Rosenqvist et al., 2008b). Hence we are able to compare our simulation results to the ones already obtained (Rosenqvist et al., 2008a). The two selected events are almost perfect mirrors of each other as the upstream parameters are steady and similar in magnitude, while there is a difference is the IMF y-component; yet the Cluster crossings occur within the same area of the magnetopause in both events $\left(x=4.4 R_{\mathrm{E}}\right.$ and $\theta_{\text {Cluster }}=45^{\circ}$ for 16 January 2001 , and $x=3.5 R_{\mathrm{E}}$ and 
$\theta_{\text {Cluster }}=36^{\circ}$ for 26 January 2001). The paper is organized as follows: first, we briefly review the methodology for inferring the energy transfer from the global MHD simulation as well as from Cluster observations. Second, we investigate the two Cluster magnetopause crossings in detail and present the performed simulations. Finally, we compare the simulation results on the energy transfer to those obtained from Cluster observations, and end the paper with discussion and conclusions. Overall, GSE coordinates are used in this paper.

\section{Methodology}

\subsection{GUMICS-4}

GUMICS-4 (Janhunen, 1996) is a state-of-the-art global MHD simulation that solves the fully conservative MHD equations within the the simulation box extending from $+32 R_{\mathrm{E}}$ to $-224 R_{\mathrm{E}}$ in $\mathrm{x}$-direction and $\pm 64 R_{\mathrm{E}}$ in the yzdirections. The magnetospheric domain is coupled with an electrostatic ionosphere: The magnetosphere determines the field-aligned currents and electron precipitation, which are given as boundary conditions to the ionospheric simulation domain. The field-aligned currents and the conductivity pattern resulting from precipitation and solar irradiation are used to determine the electric potential, which is given back to the magnetosphere, where it is used as an ionospheric boundary condition. Solar wind density, velocity, temperature and magnetic field are introduced as an input to the code at the sunward wall of the simulation box, while a variety of quantities are given as an output of the computation in space and time. GUMICS-4 uses a cell-by-cell adaptive grid, where the cells are divided into two at places with large spatial gradients.

Palmroth et al. (2003) introduced a method with which the global energy transfer can be investigated using the GUMICS-4 simulation. The method first identifies the magnetopause boundary, and then computes the simulation total energy flux perpendicular to the surface and defines this as the transferred energy. The GUMICS-4 magnetopause surface coincides with the statistical magnetopause location (Shue et al., 1997, 1998), and the method has also been found to work in other simulation runs (Shukhtina et al., 2009) using the OpenGGCM code (e.g., Raeder, 2003).

The total energy perpendicular to the magnetopause boundary is defined as the portion of energy through the magnetopause as

$$
P_{\mathrm{mp}}=\int_{A} \boldsymbol{K} \cdot \boldsymbol{n} d A
$$

where $\boldsymbol{K}$ is the total energy flux (kinetic + thermal + electromagnetic) in the GUMICS-4 simulation determined at the surface of the magnetopause, $\boldsymbol{n}$ is the unit normal vector of the surface pointing outwards, and $d A$ is the area of the surface element. In this paper, the general term "energy trans- fer" refers to Eq. (1). The computation requires that the surface is identified for each time instant, and the integration proceeds from the nose to $-30 R_{\mathrm{E}}$ in the tail. The magnetopause can be divided in smaller integration domains to study the spatial distribution of energy transfer, and one convenient way to do this is given by

$P_{A Z}(\Delta \phi)=\int_{\Delta \phi} \int_{x=\text { nose }}^{-30} \boldsymbol{K} \cdot \boldsymbol{n} d A(\phi, x)$,

where the integration is carried out from nose to the $-30 R_{\mathrm{E}}$ in sectors $\Delta \phi$ that are defined similarly as the IMF clock angle (zero in the north, $180^{\circ}$ in the south). For example, the energy transfer spatial distribution on the magnetopause in Fig. 1 is illustrated using Eq. (2) in 6 azimuthal bins $(\Delta \phi=$ $60^{\circ}$ ), and shown as polar histograms for the prevailing clock angle.

Laitinen et al. $(2006,2007)$ introduced a method to evaluate the magnetopause dynamo and reconnection powers at the magnetopause from the GUMICS-4 simulation. They computed the "energy conversion surface density", given by

$P_{\mathrm{ec}}=-\int_{-l_{1}}^{l_{2}} \nabla \cdot S d l$

where the subscript "ec" denotes energy conversion, $S$ is the Poynting vector, and the integration is carried out along the magnetopause normal through the magnetopause layer from $-l_{1}$ to $l_{2}$. Essentially, Eq. (3) computes how much magnetic energy is destroyed in the dayside reconnection region and how much magnetic energy is generated within the lobe dynamo converting the solar wind kinetic energy into magnetic energy. In this paper, a general term "energy conversion" in simulation refers to Eq. (3).

\subsection{Cluster instruments and methods}

In a time-independent case, a straightforward calculation shows that

$-\nabla \cdot \boldsymbol{S}=\boldsymbol{E} \cdot \boldsymbol{J}=\boldsymbol{J} \times \boldsymbol{B} \cdot \boldsymbol{v}$

where $\boldsymbol{E}$ is the electric field, $\boldsymbol{J}$ is current density, $\boldsymbol{B}$ is magnetic field, and $\boldsymbol{v}$ is plasma velocity. Using Eq. (4), it is possible to compute the energy conversion from spacecraft observations during a magnetopause crossing (Rosenqvist et al., 2006). Now, the integration length $d l$ is converted into $d l=\left|v_{\mathrm{mp}}\right| d t$, where the $v_{\mathrm{mp}}$ is the magnetopause velocity with respect to the spacecraft and the $d t$ is the duration of the current layer crossing. Hence, the energy conversion during a magnetopause crossing is evaluated as

$Q=\int(\boldsymbol{J} \times \boldsymbol{B}) \cdot \boldsymbol{v}\left|v_{\mathrm{mp}}\right| d t$.

Notice that while $v_{\mathrm{mp}}$ is defined in the normal direction and it can hence be either positive or negative, in Eq. (5) one must 
use the absolute value of the velocity. This is because the sign of the integrand must choose the sign of the energy conversion and the integration measure $d l=\left|v_{\mathrm{mp}}\right| d t$ only decides the size of the subareas to be summed in the final integral.

In this paper, Eq. (5) is evaluated using Cluster spacecraft observations. The magnetic field and plasma velocity are directly obtained from the Flux-Gate Magnetometer (FGM, Balogh et al., 2001) and Cluster Ion Spectrometer (CIS, Rème et al., 2001). The current density is computed using the curlometer technique (Dunlop et al., 2002), where the current density is obtained from Ampère's law and the curl of the magnetic field is computed using the observed spatial gradients within the spacecraft constellation (tetrahedron). The curlometer technique gives the most reliable estimates of the current amplitude and direction in cases where the spacecraft separation is smaller than the scale length at which the current density varies, and where the tetrahedron is not elongated but equally separated (Dunlop et al., 2002).

For the velocity of magnetopause, both multi-spacecraft methods based on timing analysis as well as single spacecraft methods are available. The relative timing of the four spacecraft observations can be used in determining the velocity and orientation of any discontinuity. Here we use constant velocity approach (CVA) assuming that the magnetopause moves at a constant speed during the constellation fly-by. The relative timings of the magnetopause crossings are found by correlating similar structures, and the orientation and velocity of the discontinuity are then computed from the timings (Dunlop and Woodward, 1998). For the single-spacecraft methods, Sonnerup et al. (2006) introduced a generic residue analysis (GRA) method, where classical conservation laws are used to determine the orientation and motion of a plasma discontinuity. The method includes conservation laws for mass, momentum, total energy, entropy, magnetic flux, and electric charge, and gives results for each conservation law. The optimal value for the orientation and motion of the discontinuity is obtained by weighting.

\section{Event descriptions}

\subsection{Upstream conditions}

Figure 2 presents the upstream conditions for the two selected events. Advanced Composition Explorer (ACE) solar wind Level 2 data are presented for the periods of 16 January 2001 (left panels) and 26 January 2001 (right panels), and a delay of $71 \mathrm{~min}$ and $69 \mathrm{~min}$ from the ACE position to $15 R_{\mathrm{E}}$ is added, respectively. The IMF observations are recorded by the magnetic field instrument (MAG) (Smith et al., 1998), while the solar wind density and velocity are determined by the Solar Wind Electron Proton Alpha Monitor (SWEPAM) instrument (McComas et al., 1998). The vertical lines denote the Cluster magnetopause crossings examined in this paper. Both crossings occur during relatively steady solar wind, and hence the exact determination of the delays added to the ACE recordings is not crucially important. The IMF intensity and solar wind density and velocity in the two events are almost identical. The significant difference during the events is that the IMF y-component is almost as much positive during the Cluster magnetopause crossing on 16 January as it is negative during the crossing at 26 January, making the clock angle $\theta$ during the events almost symmetric with respect to due south $\left(\sim 166^{\circ}\right.$ and $\left.224^{\circ}\right)$. Furthermore, during 26 January, the IMF is steadily southward for several hours prior to the time of interest, while on 16 January the IMF is northward for several hours prior to the Cluster magnetopause crossing. The $\epsilon$ parameter computed from the upstream parameters in the events is the same, $\sim 200 \mathrm{GW}$ at the times of the magnetopause crossings. As will be shown later, the time period during which the IMF is southward prior to the events is sufficiently long so that the energy transfer distribution has had time to develop at the magnetopause.

\subsection{Magnetopause crossing on 16 January 2001}

Figure 3 presents the first Cluster (spacecraft 1) magnetopause crossing at $[4.4,9.2,9.3] R_{\mathrm{E}}$ investigated in this paper. The panels (a)-(c) of Fig. 3 show the overall picture of the time period around the magnetopause crossing, representing the CIS omnidirectional proton energy spectrogram, the CIS density, and the CIS GSE velocity components from 23:00 UT until midnight. Just after 23:00 UT, Cluster observed the high energy population of the magnetosphere, while at the end of the presented period near midnight the dense magnetosheath low energy population was observed. The data show several crossings of the magnetopause, of which some are partial showing mixed populations of magnetosheath-like and magnetospheric plasma (e.g., at 23:23 UT). At 23:19 UT a full crossing occurs, during which the spacecraft passes from the magnetosphere proper into the magnetosheath proper. After 23:23 UT, the energy spectrogram shows that the spacecraft encountered the magnetopause vicinity several times. During each of these encounters, the plasma velocities increased (especially in the $v_{\mathrm{z}}$ component shown in blue). This indicates a rather stationary high speed plasma stream near the magnetopause, indicating that the structure of the magnetopause during the plotted period is rather stationary, while the magnetopause does move towards and away from the spacecraft quite a bit.

Figure $3 \mathrm{~d}-\mathrm{i}$ are blow-ups of the period marked with black lines in Fig. 3a-c, representing 18 min worth of data. Quantities needed to determine $Q$ are shown in the plot: the magnetic field (Fig. 3d) and plasma velocity components (Fig. 3e), as well as the current density components determined by the curlometer technique (Fig. 3f-h). During January 2001 , the spacecraft separation was sufficiently small to allow accurate determination of $\boldsymbol{J}$, and in Fig. 3i we plot the ratio of magnetic field divergence over the magnitude of curl. The curlometer gives reliable estimates on the current density 


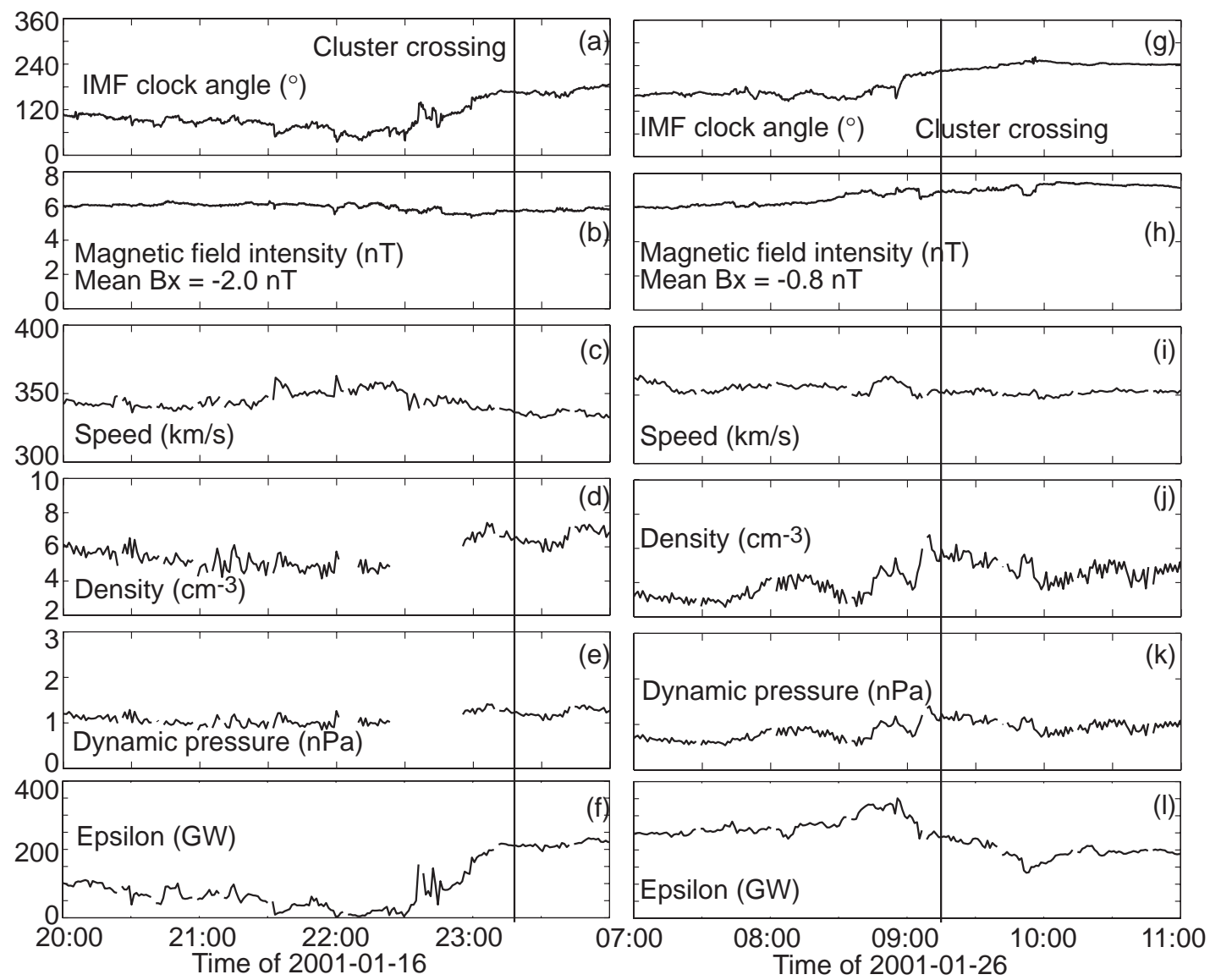

Fig. 2. Four hours worth of Advanced Composition Explorer (ACE) solar wind observations on 16 January 2001 (left panels), and on 26 January 2001 (right panels). A delay of $1 \mathrm{~h} 11 \mathrm{~min}$ and $1 \mathrm{~h} 9 \mathrm{~min}$ from ACE position to $+15 R_{\mathrm{E}}$ has been added, respectively. (a) and (g) IMF clock angle in the yz-plane, (b) and (h) magnetic field intensity, (c) and (i) solar wind speed, (d) and (j) solar wind density, (e) and (k) dynamic pressure, and (f) and (l) $\epsilon$ parameter computed using the solar wind parameters. The vertical lines denote the Cluster magnetopause crossings on each day.

when this ratio is smaller than 0.5 (Dunlop et al., 2002). The period from 23:12 UT until 23:19 UT shows positive $B_{Z}$ component, while $B_{\mathrm{X}}$ is negative, indicating that Cluster was crossing the dayside magnetopause equatorward of the cusp. The At 23:19 UT, an anti-sunward, duskward and northward current is observed, and the magnetic field rotates reaching values of the magnetosheath magnetic field. The curlometer quality factor in Fig. $3 \mathrm{~g}$ shows that except for a few points, the current estimate is reliable.

Next, we estimate the magnetopause normal and velocity for the 16 January event. The first block of Table 1 shows the results of the single-spacecraft analysis of the magnetopause normal, de Hoffman-Teller velocity, and magnetopause velocity in the normal direction. The largest ratio of the intermediate and normal eigenvalues is given by the MVAB method. The velocity of the magnetopause in the normal direction is around $-20 \mathrm{~km} \mathrm{~s}^{-1}$ for the methods using magnetic field records. Since the spacecraft velocity is negligible compared to the magnetopause velocity, the magnetopause moves inward over the spacecraft during this outbound crossing; i.e., the velocity direction is opposite to the outward pointing normal vector, explaining the negative sign in the magnetopause velocity. We also performed the CVA analysis for the magnetopause crossing using the magnetic field $L$ component in the boundary layer frame (using the MVAB normal from spacecraft 1) from all four spacecraft around the 23:19 UT. The results for the multi-spacecraft analysis are given in the second block of Table 1. The multi-spacecraft analysis is consistent with the MVAB analysis, suggesting that the magnetopause velocity during the event is around $-30 \mathrm{~km} \mathrm{~s}^{-1}$. We use both these values in the rest of the paper.

\subsection{Magnetopause crossing on 26 January 2001}

The second interval of interest occurred in the morning of 26 January 2001. This interval is extensively studied previously as it includes several consecutive magnetopause crossings over a period of almost three hours (Bosqued et al., 2001). 


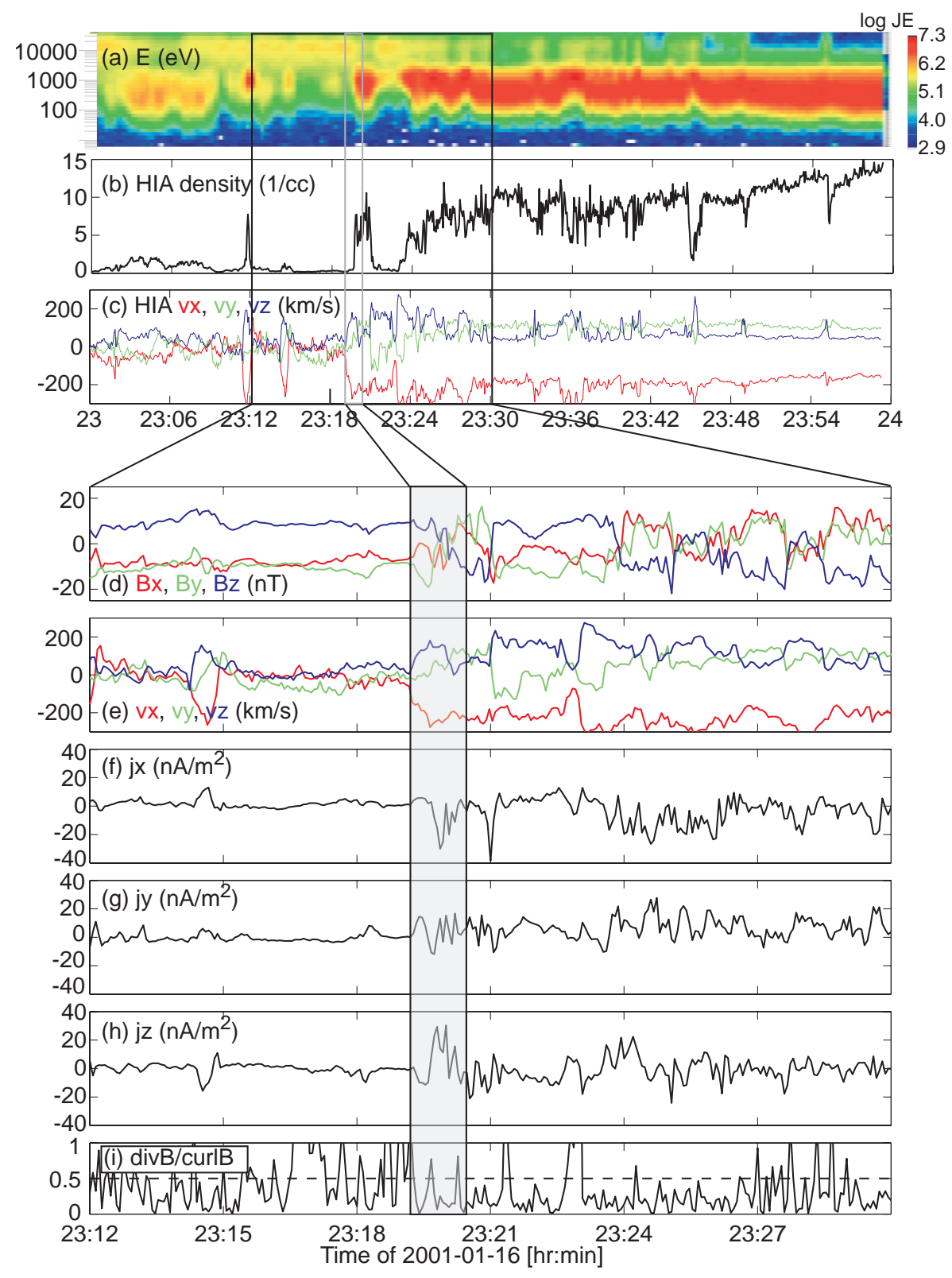

Fig. 3. Cluster spacecraft 1 observations on 16 January 2001. (a) Omnidirectional proton energy spectrogram, (b) density and (c) velocity GSE components ( $\mathrm{x}-, \mathrm{y}$ - and z-components on red, green and blue, respectively) from CIS/HIA. The black vertical lines indicate a time period for which the panels (d)-(i) are presented: (d) Magnetic field GSE components from FGM, (e) velocity of plasma (CIS), (f)-(h) x-, $\mathrm{y}$ - and z-components of current density (curlometer), and (i) the curlometer quality factor. The gray rectangle shows the exact time period of the crossing in question.

Even though in January 2001 the Cluster tetrahedron is quite elongated, (Dunlop et al., 2002) used the event as an example of a case where the current density can still be accurately determined using the curlometer technique. Figure 4 presents the curlometer current density components, the curlometer quality factor and the magnitude of the magnetic field from 09:00 UT until 12:00 UT. During the plotted period the direction of the current is northward, duskward, and tailward at most of the magnetopause crossings, indicating that the mag- netopause current is stable and consistent with the expected Chapman-Ferraro current direction. While (Rosenqvist et al., 2008a,b) chose the event at 10:30 UT, we choose the crossing taking place at 09:15 UT. This is because during that crossing the magnetopause current vector shows the typical northward signature also observed during most other crossings, while during 10:30 UT the current is atypically southward. 
Table 1. Magnetopause normal and velocity analysis for 16 January 2001; time interval 23:18:48 UT to 23:20:28 UT. Ratio is the ratio of intermediate and normal eigenvalue given by the analysis, $\boldsymbol{v}_{\mathrm{HT}}$ is the de Hoffman Teller velocity, and $\boldsymbol{v}_{\mathrm{HT}} \cdot \boldsymbol{n}$ gives the magnetopause velocity in the normal direction.

\begin{tabular}{lrccr}
\hline Method & Ratio & $\boldsymbol{v}_{\mathrm{HT}}$ & Normal & $\boldsymbol{v}_{\mathrm{HT}} \cdot \boldsymbol{n}(\mathrm{km} \mathrm{s})$ \\
\hline Minimum variance (MVAB) & 11.4 & $(-186.5,-1.6,116.3)$ & $(0.570 .310 .76)$ & -17.9 \\
Minimum Faraday residue (MFR) & 10.2 & $(-145.1,84.4,40.7)$ & $(0.550 .330 .77)$ & -20.5 \\
Minimum mass flux residue (MMR) & 4.8 & $(-228.6,103.0,84.3)$ & $(0.930 .090 .36)$ & -171.8 \\
Minimum entropy residue (MER) & 4.6 & $(-229.5,103.4,84.3)$ & $(0.930 .090 .37)$ & -171.6 \\
Combined (MVAB, MFR, MMR, MER) & 3.4 & $(-193.4,59.0,86.3)$ & $(0.740 .270 .62)$ & -72.6 \\
\hline Constant velocity analysis (CVA) & & & $(0.660 .320 .67)$ & -24.1 \\
\hline
\end{tabular}

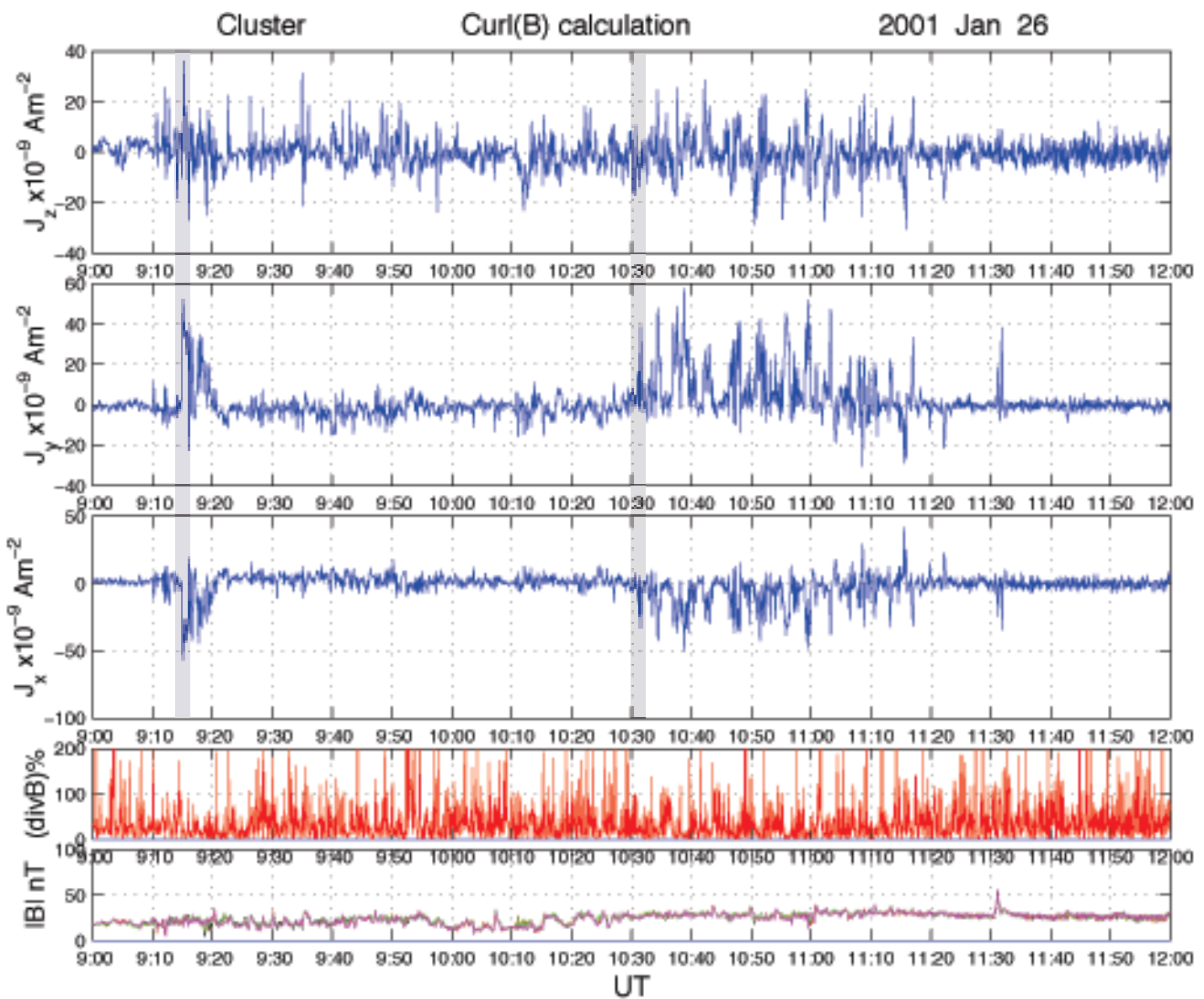

Fig. 4. The current density inferred using the curlometer technique, from 09:00 UT until 12:00 UT at 26 January 2001. Highlighted in grey are two magnetopause crossing, at 09:15 UT and 10:30 UT.

Figure 5a-c present one hour of data on 26 January 2001, from Cluster spacecraft 1 at approximately [3.5 6.7 9.1] $R_{\mathrm{E}}$, in the same format as in Fig. 3. According to Bosqued et al. (2001), the core magnetosheath population is observed at 09:17 UT (after the second gray vertical line in Fig. 5ac). The transition from the magnetosphere into the core magnetosheath population occurs through a boundary layer, where mixed populations of magnetosheath-like and magnetospheric populations are observed. After 09:14 UT, the spacecraft traverses through the boundary layer into the sheath. The presented period includes many partial crossings or skimmings of the Earthward edge of the boundary layer, during which high-velocity plasma jets oriented roughly parallel to the magnetopause are observed Bosqued et al. (2001). 
These observations indicate that the jets are associated with the structure of the boundary layer during the event.

Figure $5 \mathrm{~d}-\mathrm{i}$ shows $18 \mathrm{~min}$ worth of data around the magnetopause crossing in the same format as in Fig. $3 \mathrm{~d}-\mathrm{i}$. The period from 09:06 UT until 09:11 UT is associated with almost zero $B_{\mathrm{x}}$, and negative $B_{\mathrm{z}}$, indicating a dayside crossing equatorward of the cusp. At about 09:15 UT, the magnetic field rotates, the velocity components show a distinct change, and the current density shows an anti-sunward, duskward and northward increase consistent with the Chapman-Ferraro current direction circulating the cusps in the post-noon sector. Notice that during most of the skimmings of the boundary layer, such as at 09:23 UT, the current direction is antisunward, dawnward, and northward. This current direction is parallel to the $\boldsymbol{B} \times \boldsymbol{v}$ electric field, and hence we argue that the current signature observed during these periods is not the Chapman-Ferraro system, but is associated with the high-velocity jets.

We performed a similar single spacecraft and multi spacecraft boundary orientation and velocity analysis as for the 16 January case. The results are presented in Table 2. As the crossing does not occur from the magnetosphere proper into the sheath proper, the quality of the results are not as good as in the 16 January case. The MVAB method yields again the largest eigenvalue ratio. The CVA timing analysis is difficult as all four spacecraft do not cross the entire magnetopause layer. However, we performed the timing analysis using the first clear magnetosphere-to-boundary layer magnetic structure, and obtained a value of $-58 \mathrm{~km} \mathrm{~s}^{-1}$ for the magnetopause velocity. The value is similar to the one found in Bosqued et al. (2001), who obtained $-40 \mathrm{~km} \mathrm{~s}^{-1}$ using both single- and multi-spacecraft methods later during the same day, at 10:30 UT. As the duration of the 09:15 UT crossing is also similar to the 10:30 UT crossing, we use in the rest of the analysis the value $-40 \mathrm{~km} \mathrm{~s}^{-1}$, agreeing sufficiently well with the CVA and MVAB.

\section{GUMICS runs}

GUMICS-4 was executed with the solar wind input from the periods given in Fig. 2. The smallest grid spacing in the simulation runs is $0.25 R_{\mathrm{E}}$, ensuring a sharp boundary at the magnetopause. Due to the code setup where the solar wind magnetic field needs to be divergenceless, solar wind $B_{\mathrm{x}}$ was set to zero. The dipole tilt angle in both runs is set to zero, otherwise the code setup is typical that has been used in several event simulations (e.g., Palmroth et al., 2003). There are indications that the IMF $B_{\mathrm{x}}$ and the tilt angle affect the reconnection line location (e.g., Trenchi et al., 2008) and hence the approximations for the tilt angle and the IMF $B_{\mathrm{x}}$ might be invalid in investigations of the load and generator areas. However, as the negative tilt in January and the negative IMF $B_{\mathrm{X}}$ shift the reconnection line into opposite directions, and the negative tilt has only a slight effect in the North- ern Hemisphere where the Cluster crossings occur (Palmroth et al., 2011), the assumptions concerning the tilt and IMF $B_{\mathrm{x}}$ are valid. Figure 6 illustrates the Cluster orbits on 16 January (left panels) and 26 January (right panels) overlaid with GUMICS-4 reproduction of the plasma density for both events.

\section{Results: energy transfer and conversion on magnetopause}

Figure 7 shows the total energy computations and azimuthal energy distributions for the 16 January event. The temporal variation of the total energy transfer through the GUMICS4 magnetopause resembles that of the $\epsilon$ parameter, while the magnitudes are different. This is due to the fact that $\epsilon$ is scaled to the magnetospheric energy consumption, while the GUMICS-4 energy transfer (Eq. 1) includes all energy transferred through the surface until $x=-30 R_{\mathrm{E}}$, which is not necessarily deposited within the ionosphere or the inner magnetosphere. Therefore, Fig. 7b also shows the $\epsilon$ parameter scaled with the simulation magnetopause mean area (red) during the run instead of the traditional $4 \pi l_{0}^{2}$ scaling parameter, where $l_{0}=7 R_{\mathrm{E}}$. The vertical lines in Fig. $7 \mathrm{~b}$ denote the time instants at which we present azimuthal energy transfer distributions shown in Fig. 7c computed using Eq. (2). The $\phi$ axis at the outer circle shows the magnetopause in yz-plane looking tailward, and the energy transfer through each sector $\Delta \phi$ is given by a bar, whose size is proportional to the energy input in that sector, normalized to the outer circle $(100 \mathrm{GW})$. The black line and dot in each energy distribution shows the IMF orientation and clock angle.

The azimuthal energy transfer distributions in Fig. 7c clearly show that during southward IMF, the energy transfers through the magnetopause surface in sectors aligned with the plane of the IMF due to the Poynting flux focussing (Palmroth et al., 2003): the electromagnetic energy vector points towards the magnetopause in those locations, where the newly opened field lines are advecting tailwards, because only at those locations the magnetic field lines are at an angle with the magnetosheath velocity field allowing a nonzero Poynting flux. The field line advection in sectors aligned with the plane of the IMF is also predicted by the Cooling model (Cooling et al., 2001) used to track the flux transfer events on the magnetopause. In Fig. 7c, it is important to notice that while the rightmost distribution resembles the energy transfer distribution during the actual Cluster magnetopause crossing, the distributions are all qualitatively similar: they are all tilted in the plane of the IMF that stays between $116^{\circ}$ and $166^{\circ}$. Based on Fig. 7c, we expect a priori that the energy conversion on the Cluster magnetopause crossing will be small, as Cluster is not sampling the magnetopause in the sector of large energy transfer (see Cluster position in Fig. 6b). 


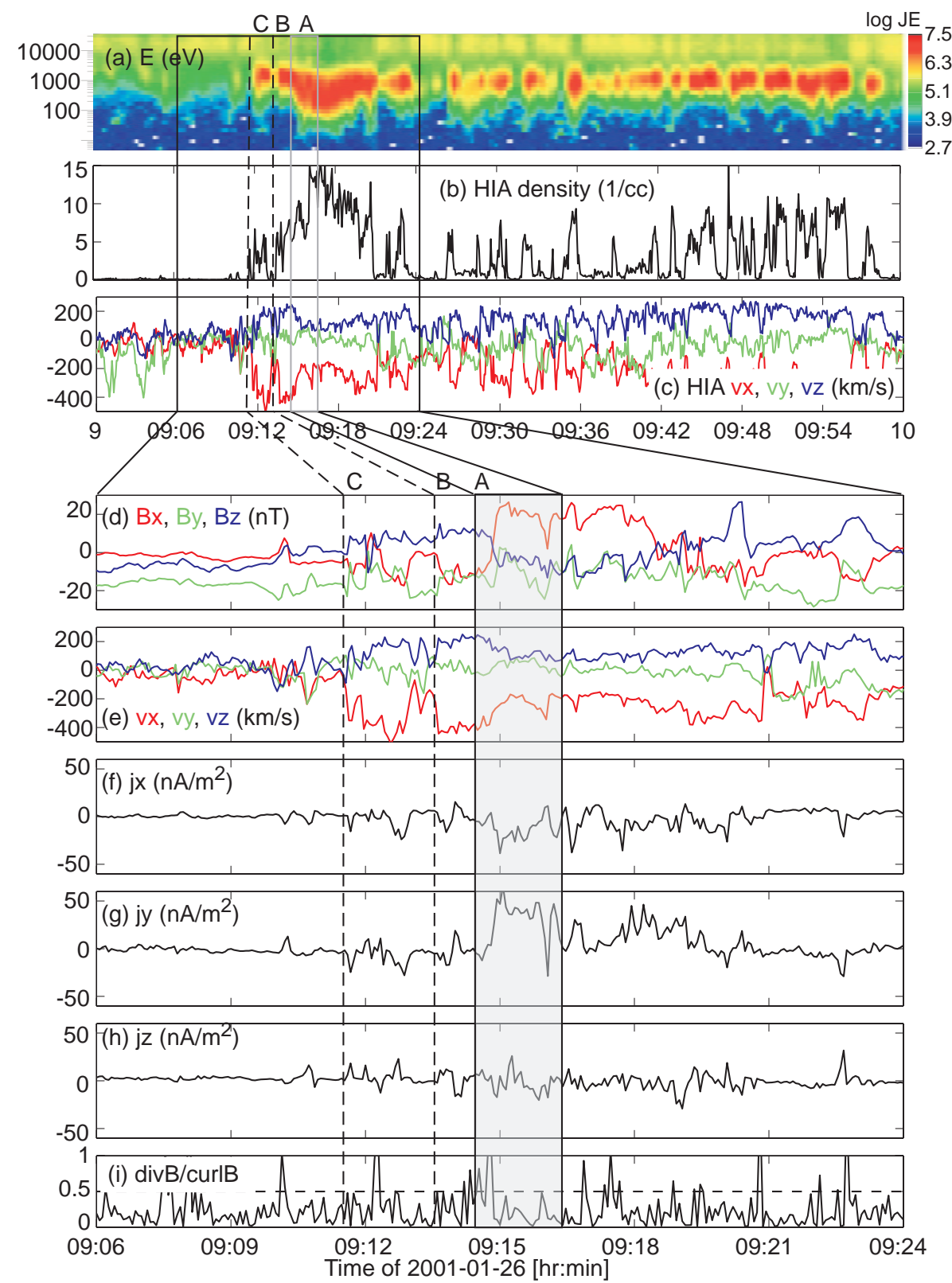

Fig. 5. Cluster spacecraft 1 observations on 26 January 2001, from spacecraft 1 . (a) Omnidirectional proton energy spectrogram, (b) density and (c) velocity GSE components (x-, y- and z-components on red, green and blue, respectively) from CIS/HIA. The black vertical lines indicate a time period for which the panels (d)-(i) are presented: (d) magnetic field GSE components from FGM, (e) velocity of plasma (CIS), (f)-(h) x-, y- and z-components of current density (curlometer), and i) the curlometer quality factor. The gray rectangle, vertical dashed lines and letters A, B and C refer to Table 3 .

Figure 8 shows the $\epsilon$ parameter, total energy transfer in GUMICS-4 as well as the azimuthal energy transfer distributions for 26 January, in the same format as in Fig. 7. The vertical lines are now showing the time instants separated by $10 \mathrm{~min}$, and centered by the Cluster magnetopause crossing that took place about 09:15 UT. Again, the energy transfers in the plane of the IMF, and the distributions in Fig. 8c stay qualitatively similar at an after 09:15 UT, although the amount of the transferred energy varies slightly. The scaled $\epsilon$ is again in good accordance with the simulation energy input through the magnetopause. Figure $6 \mathrm{~d}$ shows the Cluster orbit for the denoted time instants, and now the spacecraft cross the magnetopause in a sector, where also a large amount of energy is transferring. Hence, we again expect that Cluster observes a large amount of energy conversion during the magnetopause crossing. 
Table 2. Magnetopause normal and velocity analysis for 26 January 2001; time interval 09:14:30 UT to 09:15:20 UT. The format is the same as in Table 1.

\begin{tabular}{lrccr}
\hline Method & Ratio & $\boldsymbol{v}_{\mathrm{HT}}$ & Normal & $\boldsymbol{v}_{\mathrm{HT}} \cdot \boldsymbol{n}\left(\mathrm{km} \mathrm{s}^{-1}\right)$ \\
\hline Minimum variance (MVAB) & 3.3 & $(-321.668 .9145 .7)$ & $(0.45-0.200 .87)$ & -33.3 \\
Minimum Faraday residue (MFR) & 2.4 & $(-73.9161 .441 .3)$ & $(0.42-0.010 .91)$ & 5.2 \\
Minimum mass flux residue (MMR) & 1.0 & $(-36.182 .725 .4)$ & $(0.54-0.830 .16)$ & -83.9 \\
Minimum entropy residue (MER) & 1.1 & $(-21.087 .816 .1)$ & $(0.54-0.820 .15)$ & -81.4 \\
Combined (MVAB, MFR, MMR, MER) & 2.5 & $(-253.490 .9116 .9)$ & $(0.44-0.120 .89)$ & -19.0 \\
\hline Constant velocity analysis (CVA) & & & $(0.600 .330 .73)$ & -58.2 \\
\hline
\end{tabular}
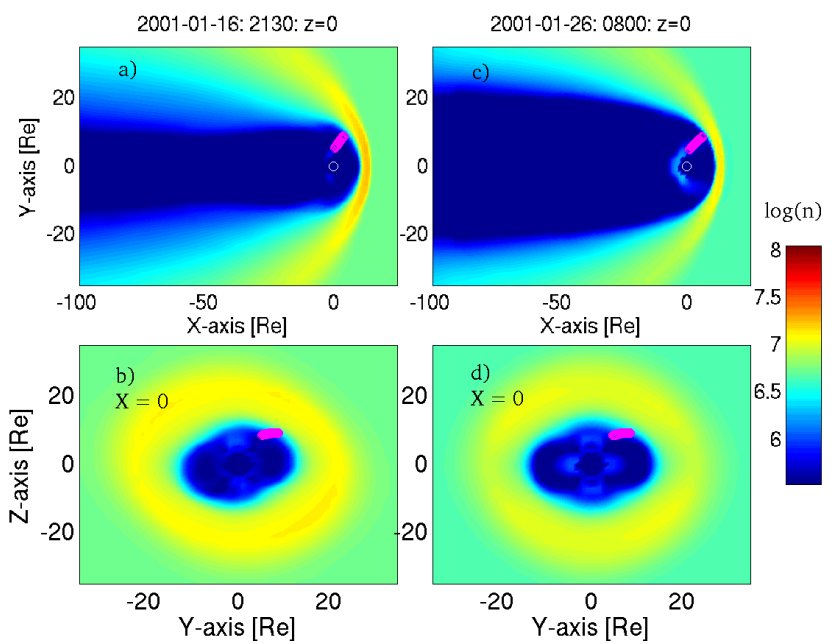

Fig. 6. Cluster spacecraft positions (magenta circles) on 16 January (panels a and b), and 26 January (panels $\mathbf{c}$ and $\mathbf{d}$ ) during the period presented in Fig. 2. The colorcoding is the GUMICS-4 reproduction of logarithm of plasma density during the two events. Panels (a) and (c) are depicted in xy-plane at $z=0$, whereas panels (b) and (d) are those for yz-plane at $x=0$.

Figure 9 shows the results of the detailed comparison between the GUMICS-4 simulation against the Cluster estimate of the energy conversion, calculated using the data from times highlighted with gray in Figs. 3 and 5. The left (right) panels are again for 16 January (26 January) events. The top row shows the GUMICS-4 energy conversion computed using Eq. (3), while the second row gives the energy transfer using Eq. (1). The magnetopause is viewed from the front looking tailwards. The magenta dots give the Cluster position in each event at the given time. The GUMICS-4 results on the energy conversion and transfer at the Cluster position are given in the respective legends of Fig. 9a-b and $9 d-$ e. The GUMICS-4 results for 16 January are evaluated at 23:15 UT, and 09:15 UT on 26 January. The energy transfer distributions depicted in Fig. 9b and 9e are almost as much tilted with respect of due south and show almost similar magnitudes of energy transfer as the other solar wind conditions
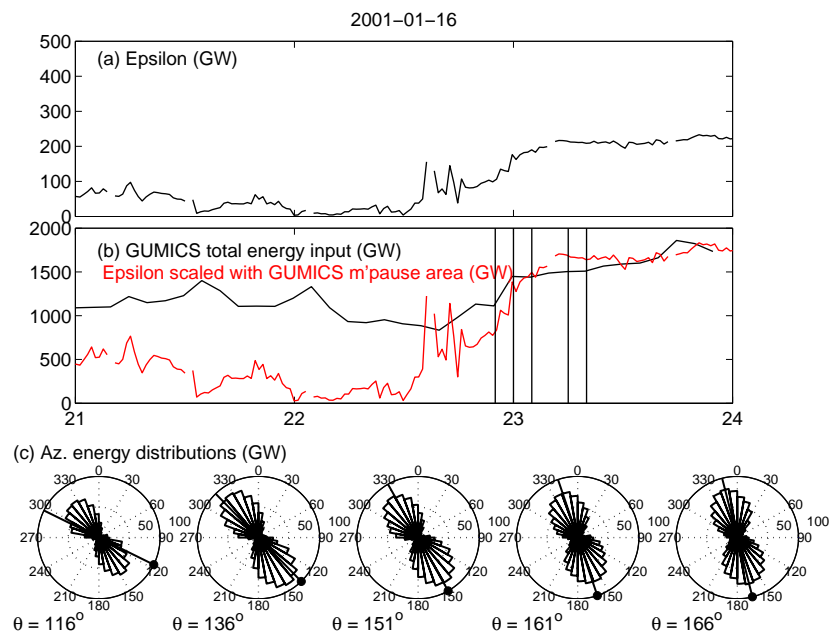

Fig. 7. (a) The $\epsilon$ parameter for 16 January event, delayed to $+15 R_{\mathrm{E}}$ using a delay $1 \mathrm{~h} 11 \mathrm{~min}$. (b) Total energy transfer through the magnetopause in the GUMICS-4 simulation against time in the 16 January event (black) and $\epsilon$ parameter scaled with the simulation magnetopause area (red). Vertical lines denote the times at which the instantaneous energy transfer distributions in (c) are given. The size of the bar in panels (c) gives the portion of energy transfer in the yz-plane integrated from the nose to $-30 R_{\mathrm{E}}$. The bar size is normalized to the outer circe $(100 \mathrm{GW})$, and the IMF orientation is given by the black line, with the filled dot referring to the clock angle given in the bottom left legend of each distribution.

are similar. The bottom row gives the Cluster estimate of the energy conversion $Q$ using Eq. (5) in the two events. The integral of the energy conversion through the magnetopause is computed as a cumulative sum, and hence the final value of the plotted curve given in the legend of Fig. $9 \mathrm{c}, \mathrm{f}$ is to be compared with the simulation results. The Cluster estimate for 16 January is computed using two values for the magnetopause velocity: $20 \mathrm{~km} \mathrm{~s}^{-1}$ (black) and $30 \mathrm{~km} \mathrm{~s}^{-1}$ (red), while the value for 26 January uses $40 \mathrm{~km} \mathrm{~s}^{-1}$ found here and in Bosqued et al. (2001).

Figure 9 illustrates that on 16 January, the spatial distribution of energy conversion and transfer is tilted away from 
Table 3. Cluster estimations of the energy conversion using different time intervals and including all current density values in the calculation $\left(Q_{1}\right)$ and omitting those current density values where the curlometer quality factor is larger than $0.5\left(Q_{2}\right)$. The difference $(\%)$ tells how much $Q_{1}$ differs from $Q_{2}$.

\begin{tabular}{lrrrr}
\hline Time & $Q_{1}\left(\mu \mathrm{W} \mathrm{m}^{-2}\right)$ & $Q_{2}\left(\mu \mathrm{W} \mathrm{m}^{-2}\right)$ & $v_{\mathrm{mp}}\left(\mathrm{km} \mathrm{s}^{-1}\right)$ & Difference \\
\hline 16 January 2001 & & & & \\
\hline 23:18:36-23:20:27 UT & -8.6 & -5.3 & 20 & $38 \%$ \\
23:18:36-23:20:27 UT & -12.8 & -8.0 & 30 & $38 \%$ \\
\hline 26 January 2001 & & & \\
\hline A: 09:14:27-09:16:23 & -106.4 & -95.9 & 40 & $10 \%$ \\
B: 09:13:30-09:16:23 & -102.1 & -89.1 & 40 & $12 \%$ \\
C: 09:11:31-09:16:23 & -130.0 & -114.3 & & $12 \%$ \\
\hline
\end{tabular}
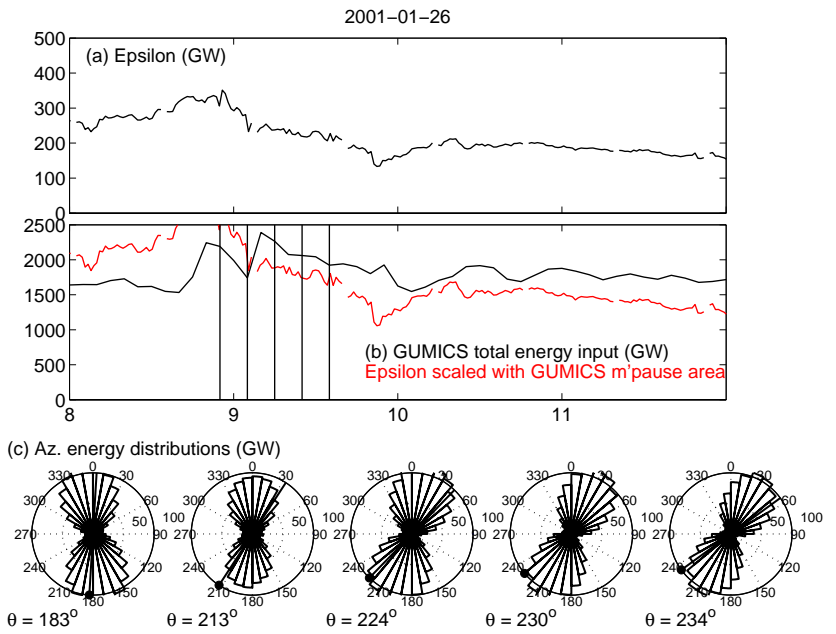

Fig. 8. (a) The $\epsilon$ parameter for 26 January event, delayed to $+15 R_{\mathrm{E}}$ using a delay $1 \mathrm{~h} 9 \mathrm{~min}$. (b) Total energy transfer through the magnetopause in the GUMICS-4 simulation against time in the 26 January event (black) and $\epsilon$ parameter scaled with the simulation magnetopause area (red). Vertical lines denote the times at which the instantaneous energy transfer distributions in (c) are given. The format of the figure is the same as Fig. 7.

the north-south direction, and occurs in the northern dawn and southern dusk sectors at the magnetopause. The Cluster crossing of the magnetopause occurs in between the load and generator regions away from the strongest energy conversion and transfer, and indeed in Fig. 9c the Cluster estimate of the energy conversion within the magnetopause current layer is small, only from -8 to $-13 \mu \mathrm{W} \mathrm{m}{ }^{-2}$. The Cluster estimate is larger than in GUMICS, but still in quantitative accordance with the simulation results: The simulation shows little energy conversion and transfer, as the conversion estimate is about $-3 \mu \mathrm{W} \mathrm{m} m^{-2}$ and transfer about $-4 \mu \mathrm{W} \mathrm{m} m^{-2}$. The pixels neighboring the Cluster crossing location give similar magnitudes, but can be of different sign. On 26 Jan- uary, however, Cluster crosses the magnetopause in a region where the simulation results indicate large energy conversion and transfer. Based on the simulation, the location of the crossing occurs well within the generator region as now the neighboring pixels show similar magnitudes and sign for energy conversion, indicating also that our initial assumptions of the tilt angle and IMF $B_{\mathrm{x}}$ are valid. The simulation estimates for the conversion and transfer are $-28 \mu \mathrm{W} \mathrm{m}{ }^{-2}$ and $-50 \mu \mathrm{W} \mathrm{m}{ }^{-2}$, respectively, lower than the Cluster estimate, which is $-106 \mu \mathrm{W} \mathrm{m}^{-2}$. In both events, the Cluster estimate of the energy conversion exceeds that of the GUMICS-4 local energy conversion by the same factor $\sim 4$.

Table 3 gives Cluster estimations of the energy conversion from the two events using different crossing parameters. The 16 January crossing is "clean", such that there is no ambiguity on the timing of the crossing, and as indicated by Fig. 3, the spacecraft traverses from the magnetospheric-like into sheath-like population rapidly without observing a boundary layer. The ambiguity within the crossing comes from the exact value of the magnetopause velocity, and the few points of possibly erroneous curlometer current density measurements. Hence, we present the $Q$ calculation using the two magnetopause velocity values as well as omitting the data points having a larger curlometer quality factor than 0.5 . The value $Q_{1}$ is hence computed using all points from the time period, but in computing the value $Q_{2}$ the points where the curlometer quality factor exceeds the 0.5 limit are set to zero. As the GUMICS-4 result was $-2.9 \mu \mathrm{W} \mathrm{m}^{-2}$, the Cluster estimate is larger by a factor of 2-3.

The 26 January event is more ambiguous in timing, as the spacecraft flies through the boundary layer and the highvelocity jets and their associated currents disturb the timing based on the current density increase. Table 3 shows the differences in estimates for $Q$ in three crossing durations (letters A, B, and C in Fig. 5). Taking into account the ambiguity associated with the timing, the curlometer, and the velocity of the magnetopause, our best estimate of the energy conversion within the magnetopause in the 26 January event is 

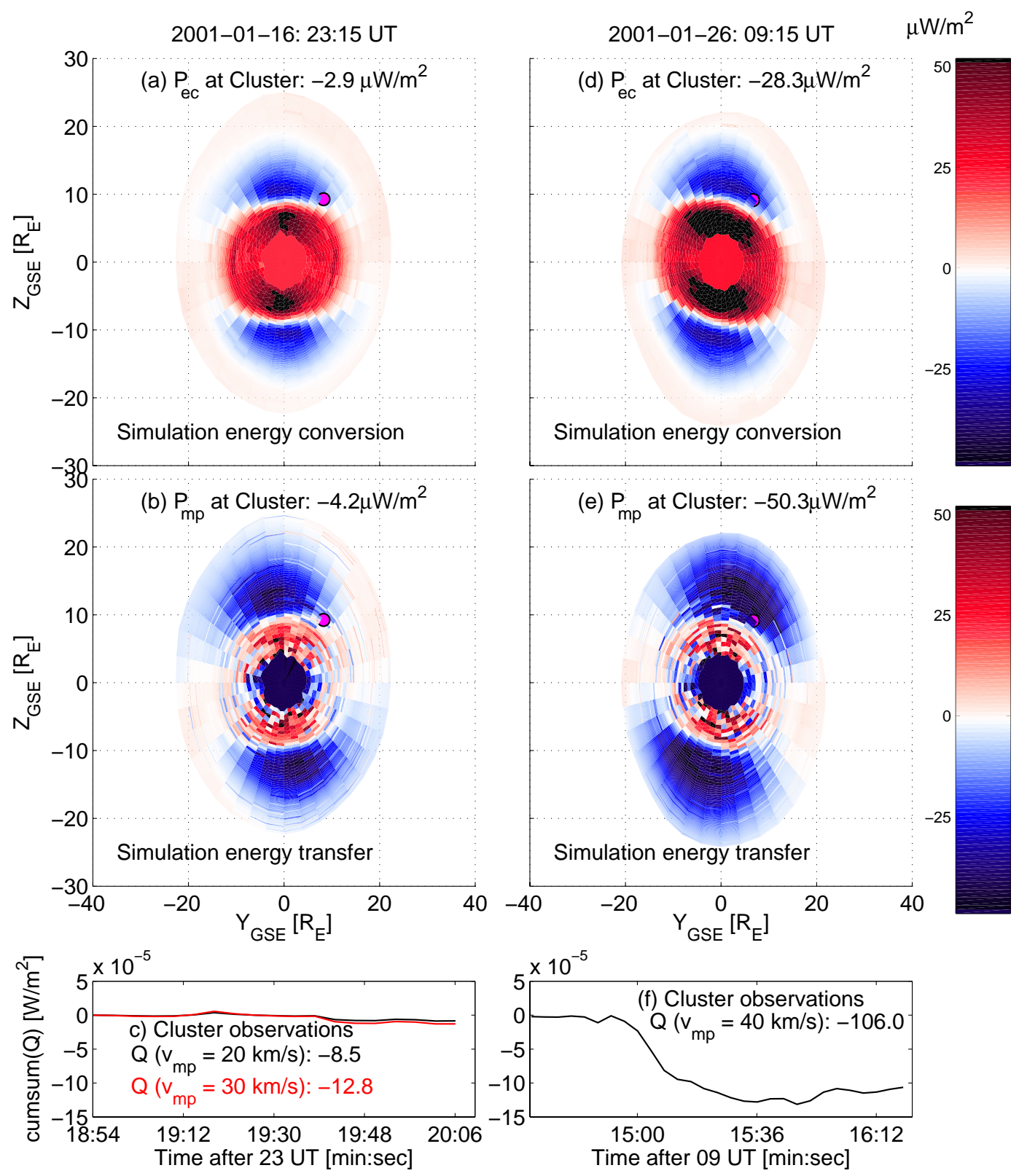

Fig. 9. Left (right) panels: results for 16 January at 23:15 UT (26 January at 09:15 UT). (a) and (d) divergence of the Poynting vector at the magnetopause surface in the GUMICS-4, looking tailwards from the front of the magnetopause. (b) and (e) total energy transfer through the magnetopause surface in the GUMICS-4. (c) and (f) Cumulative sum (representing the time evolution of the $Q$ integral) of energy conversion at Cluster orbit through the magnetopause; red (black) curve using $v_{\mathrm{mp}}=20(30) \mathrm{km} \mathrm{s}^{-1}$ for 16 January. The magenta dots in panels (a), (b), (d) and (e) show the Cluster position on the given time, and the values in the respective legends show the simulation result on Cluster position at the given time. The Cluster estimate of the integral of the energy conversion (the final value of the cumulative sum) in the magnetopause current layer is given in the legends of panels (c) and (f). All values in legends are given in $\mu \mathrm{W} \mathrm{m}^{-2}$

about $-100 \mu \mathrm{W} \mathrm{m}{ }^{-2}$, again larger by a factor of 3 compared to the GUMICS-4 local values. Hence in both events, ambiguity of the measurements explained a factor of 1 discrepancy between the measurements and the simulation results, but the same scaling factor of 2-3 was found between the Cluster observations and the simulation results.

\section{Discussion}

In this paper, our main goal is to use the simulation to verify the IMF $B_{\mathrm{y}}$ dependence of the spatial energy transfer suggested by earlier simulations (Palmroth et al., 2003). We can also take the opportunity to estimate the global energy transfer using the two local measurements to scale the simulation 
results. We find the same scaling parameter (a factor of 2-3) from both local estimates. We have also briefly reviewed the methodology developed earlier to infer the simulation energy transfer and conversion from GUMICS-4 global MHD simulation (Palmroth et al., 2003; Laitinen et al., 2006, 2007). The two methods represent two complementary viewpoints in the magnetopause energetics and they are both consequences of the dayside magnetopause reconnection. The energy transfer method tells us how much total energy (kinetic, electromagnetic and thermal) transfers across the magnetopause, while the energy conversion method yields an estimate of how much of the transferring energy is converted from one form to another, and directly evaluates the power consumed by reconnection. Hence in principle, the magnitude of energy conversion cannot exceed that of the energy transfer. The spatial variation of the transfer and conversion is not necessarily exactly the same as the integrals are different, although using primarily the same quantities. The energy conversion occurs primarily within or adjacent to the reconnection region, but energy can transfer (via Poynting flux focussing) anywhere on the surface, where open field lines exist. The energy conversion method should be comparable to the Cluster methodology (Rosenqvist et al., 2006) in a timeindependent case, as shown by Eq. (4). Time-independency is a good assumption if the magnetopause structure remains steady during the event. This is the case in both of the events discussed here.

In computing the Cluster estimate of the magnetopause energy conversion, obvious sources of errors include the determination of the current density and the magnetopause velocity. Especially the latter is a constant multiplier in Eq. (5) and order-of-magnitude errors would introduce an order of magnitude discrepancy in the final estimation. Here, we have carefully measured the velocity of the magnetopause. We have also used the best available method (curlometer) to infer the current density, and we note that the single spacecraft methods yield similar values (not shown). Hence, our current density estimate is generally reliable, while instantaneous observations include uncertainties that lead to discrepancies within the final estimate (Table 3). As witnessed during the 26 January 2001 event, the magnetopause can include local effects that are associated with boundary layer dynamics. Hence, we argue that the timing should be done as carefully as possible, so that only the large scale ChapmanFerraro current contributes to $Q$. Special care should be paid on the timing of the event if it includes spiky current density features that are not consistent with the large-scale current direction. However, one of the most important finding of this paper is that even with the best possible means to infer energy conversion (multi-spacecraft techniques, carefully selected events and stable upstream conditions) an uncertainty factor exists within the observations. Here, the final estimates include $10 \%-40 \%$ differences, which were due to timing, velocity of the magnetopause as well as momentary bad values of the curlometer. We envisage that in more dynamic events having unstable upstream conditions, the discrepancies can be larger.

The 26 January case was also one of many crossings analysed by Rosenqvist et al. (2008b,a). They $+67 \mu \mathrm{W} \mathrm{m}^{-2}$ for $Q$ at 10:30 UT and interpreted the event as being a crossing of the load region. Using the BATS-R-US global MHD simulation, Rosenqvist et al. (2008a) computed both $Q$ and $-\nabla \cdot S$ from the simulation results along the Cluster orbit. The comparison yielded favorable results only after they artificially lowered the spacecraft trajectory in the simulation towards the subsolar magnetopause. We note that in Rosenqvist et al. $(2008 \mathrm{a}, \mathrm{b})$ the a priori assumption on the load nature of the crossing was made based on the current theoretical understanding that the load exists equatorward of the cusp (Lundin and Evans, 1985). However, most importantly, the current density during the 10:30 UT event shows southward signatures, while typically the magnetopause crossings on 26 January show northward current densities (Fig. 4). Flipping the sign of the $J_{\mathrm{Z}}$ to positive at 10:30 UT flips the sign of $Q$ into negative, consistent with our findings of the 09:15 UT crossing. Since the current shows northward signatures during several of the crossings, we note that the current density direction at 09:15 UT is consistent with the global ChapmanFerraro direction, while the 10:30 UT crossing possibly includes local signatures that influence the current direction. The global simulations cannot easily reproduce local signatures, while the global pattern is reproduced on average. Indeed, Fig. 9 shows a large positive region equatorward of the generator region, and hence the artificial lowering of the spacecraft orbit in a simulation would yield a good agreement.

The current theoretical understanding states that the load region resides equatorward of the cusp, while the generator region is found poleward of the cusp. The Cluster results shown in this paper suggest that generator region can be found from the dayside magnetosphere on field lines that are equatorward of the cusp. The use of the spatially limited cusp to distinguish the load and generator regions is misleading especially for nonzero IMF y when the magnetospheric axis of symmetry is not in the noon-midnight meridian. For instance in the presented 26 January 2001 case, the IMF y is negative and hence the cusps are found from the northern pre-noon and the southern post-noon regions (Newell et al., 1989), while the Cluster crossing occurs in the northern post-noon, a large longitudinal distance away from the cusp. Instead, we argue that theoretically the accurate separator for the load and generator regions, at least for low-latitude reconnection, is the location at which the solar wind thrust force exceeds the $\boldsymbol{J} \times \boldsymbol{B}$ stress caused by the curvature of the open field line. In other words, the separator for load and generator should appear where the Alfvén velocity equals the magnetosheath velocity, and the field line is being dragged by the magnetosheath flow instead of being accelerated by reconnection. As such, this occurs tailward of the last closed field line that indeed is the cusp field line somewhere on the 
surface, and hence the cusp is a special case of this general condition. While it would be interesting to find this separator, we leave it for further study with a notion that the separator search should start by finding a location where the acceleration fields caused by the magnetosheath flow and the $\boldsymbol{J} \times \boldsymbol{B}$ force are in balance. This also introduces interesting avenues for further studies of the magnetopause energy transfer as it suggests that the load and generator regions (their sizes and possibly also their intensities) can be dependent on the magnetosheath velocity field, which in turn is related not only to the velocity of the solar wind but also to the shock structure.

In a global simulation using a quantitative methodology the a priori assumptions are more easily formed as the outcome of the calculation is plainly visible as in Fig. 1, emphasizing the power of the approach combining the simulations with observations. When looking at the simulation data giving a full three-dimensional global picture of the two events, the Cluster estimates fall naturally in place and are almost in quantitative agreement with the simulation results. We believe that here the Cluster estimate and the simulation results validate each other: the simulations show that the large differences in the Cluster estimates is natural and due to the spatial variation of energy transfer and conversion. On the other hand, the carefully measured Cluster estimate pins down the magnitudes of the simulation results. Assuming that the comparison between in situ observations and the simulation is as good also in other parts of the magnetopause, we are able to pin down the total energy transfer during the two time instants. We estimate that the total energy transferring through the magnetopause during the two events is about 1500-2000 GW, three times the value of $\epsilon$. The $\epsilon$ represents Poynting flux through a circular area of radius $l_{0}$, where the radius is used to scale the energy input to equal the magnetospheric output. To make the simulation results more directly comparable with $\epsilon$, we have scaled the $\epsilon$ again with the magnetopause mean area during the events, as measured from the simulation. Our results show that the local values in the simulation are underestimated by a factor of probably $2-3$, while the scaled $\epsilon$ is in quantitative agreement with the simulation energy input. This indicates that $\epsilon$ is underestimated based on the evidence of this paper. In accordance, Koskinen and Tanskanen (2001) also suggested in a broad review of the $\epsilon$ parameter that $\epsilon$ should be scaled up by a factor of $1.5-2$.

\section{Summary and conclusions}

We conclude that the GUMICS-4 simulation results are in good agreement with the Cluster observations in these two cases. The magnitude of energy conversion in the simulation, obtained by means that are directly comparable to the methodology using the Cluster observations, is around $30 \%$ of the Cluster estimate during both events, without any assumptions made on the magnetopause velocity or the relative location of the Cluster magnetopause crossing within the code. The simulation energy transfer values are around 50\% of the Cluster estimate. However, as the observations also include uncertainties, we conclude that using the present grid resolution and within the global framework the comparison is as perfect as it can be.

Our main findings in this paper are the following:

1. Cluster observations verify the simulation results on the IMF $B_{\mathrm{y}}$ dependence of the energy transfer on the magnetopause.

2. To estimate global energy transfer, one should only take current layers being part of the Chapman-Ferraro system.

3. The separator for load and generator should appear where the Alfvén velocity equals the magnetosheath velocity, and the field line is being dragged by the magnetosheath flow instead of being accelerated by reconnection.

4. The amount of energy conversion and transfer in GUMICS-4 agrees well with Cluster observations during the presented events, even though shows probably a factor of 2-3 lower values, however, also the Cluster estimate of $Q$ includes ambiguities.

5. The combined results from the simulation and Cluster observations suggest that the $\epsilon$ parameter is underestimated by a factor of $2-3$.

Acknowledgements. The Cluster data have been retrieved from the Cluster Active Archive (CAA), and we thank Harri Laakso and team for maintaining the system. ACE spacecraft recordings are retrieved from the ACE homepage (http://www.srl.caltech.edu/ACE/). We thank the ACE MAG and SWEPAM PI's C. W. Smith and D. J. McComas for distributing the Level 2 data freely in the internet. We thank the CDAWeb facility for distributing the data. The research leading to these results has received funding from the European Research Council under the European Community's Seventh Framework Programme (FP7/2007-2013)/ERC Starting Grant agreement number 200141-QuESpace. The work of MP is supported by the Academy of Finland. MWD is partly supported by Chinese Academy of Sciences (CAS) visiting Professorship for senior international scientists grant no. 2009S1-54 and the Specialized Research Fund for State Key Laboratories of the CAS.

Guest Editor M. Taylor thanks M. Liemohn and A. R. Retinò for their help in evaluating this paper.

\section{References}

Akasofu, S.-I.: Energy coupling between the solar wind and the magnetosphere, Space Sci. Rev., 28, 121-190, 1981.

Axford, W. I. and Hines, C. O.: A unifying theory of high-latitude geophysical phenomena and geomagnetic storms, Can. J. Phys. 39, 1433-1464, 1961

Balogh, A., Carr, C. M., Acuña, M. H., Dunlop, M. W., Beek, T. J., Brown, P., Fornacon, K.-H., Georgescu, E., Glassmeier, K.H., Harris, J., Musmann, G., Oddy, T., and Schwingenschuh, K.: 
The Cluster Magnetic Field Investigation: overview of in-flight performance and initial results, Ann. Geophys., 19, 1207-1217, doi:10.5194/angeo-19-1207-2001, 2001.

Bosqued, J. M., Phan, T. D., Dandouras, I., Escoubet, C. P., Rème, H., Balogh, A., Dunlop, M. W., Alcaydé, D., Amata, E., Bavassano-Cattaneo, M.-B., Bruno, R., Carlson, C., DiLellis, A. M., Eliasson, L., Formisano, V., Kistler, L. M., Klecker, B., Korth, A., Kucharek, H., Lundin, R., McCarthy, M., McFadden, J. P., Möbius, E., Parks, G. K., and Sauvaud, J.-A.: Cluster observations of the high-latitude magnetopause and cusp: initial results from the CIS ion instruments, Ann. Geophys., 19, 1545-1566, doi:10.5194/angeo-19-1545-2001, 2001.

Boyle, C. B., Reiff, P. H., and Hairston, M. R.: Empirical polar cap potentials, J. Geophys. Res., 102(A1), 111-125, doi:10.1029/96JA01742, 1997.

Cooling, B. M. A., Owen, C. J., and Schwartz, S. J.: Role of the magnetosheath flow in determining the motion of open flux tubes, J. Geophys. Res., 106, 18764-18775, 2001.

Dungey, J. W.: Interplanetary field and the auroral zones, Phys. Res. Lett., 6, 47-48, 1961.

Dunlop, M. W. and Woodward, T. I.: Multi-spacecraft discontinuity analysis: orientation and motion, in: Analysis methods for multispacecraft data, chapter 11, 271-306, edited by: Paschmann, G. and Daly, P. W., ISSI scientific report SR-001, ESA Publications Division, The Netherlands, 1998.

Dunlop, M. W., Balogh, A., Glassmeier, K.-H., and Robert, P.: Four-point Cluster application of magnetic field analysis tools: The Curlometer, J. Geophys. Res., 107(A11), 1384, doi:10.1029/2001JA005088, 2002.

Janhunen, P.: GUMICS-3: A global ionosphere-magnetosphere coupling simulation with high ionospheric resolution, in: Proceedings of Environmental Modelling for Space-Based Applications, 18-20 September 1996, Eur. Space Agency Spec. Publ., ESA SP-392, 1996.

Koskinen, H. E. J. and Tanskanen, E. I.: Magnetospheric energy budget and the epsilon parameter, J. Geophys. Res., 107(A11), 1415, doi:10.1029/2002JA009283, 2002.

Laitinen, T. V., Janhunen, P., Pulkkinen, T. I., Palmroth, M., and Koskinen, H. E. J.: On the characterization of magnetic reconnection in global MHD simulations, Ann. Geophys., 24, 30593069, doi:10.5194/angeo-24-3059-2006, 2006.

Laitinen, T. V., Palmroth, M., Pulkkinen, T. I., Janhunen, P., and Koskinen, H. E. J.: Continuous reconnection line and pressure-dependent energy conversion on the magnetopause in a global MHD model, J. Geophys. Res., 112, A11201, doi:10.1029/2007JA012352, 2007.

Lundin, R. and Evans, D. S.: Boundary layer plasmas as a source for high-latitude, early afternoon, auroral arcs, Planet. Space Sci., 33, 1389-1406, 1985.

McComas, D. J., Bame, S. J., Barker, P., Feldman, W. C., Phillips, J. L., Riley, P., and Griffee, J. W.: Solar Wind Electron Proton Alpha Monitor (SWEPAM) for the Advanced Composition Explorer, Space Sci. Rev., 86, 563-612, 1998.

Newell, P. T., Meng, C.-I., and Sibeck, D. G.: Some low-altitude cusp dependencies on the interplanetary magnetic field, J. Geophys. Res., 94, 8921-8927, 1987.

Newell, P. T., Sotirelis, T., Liou, K., Meng, C.-I., and Rich, F. J.: A nearly universal solar wind-magnetosphere coupling function inferred from 10 magnetospheric state variables, J. Geophys. Res.,
112, A01206, doi:10.1029/2006JA012015, 2007.

Palmroth, M., Pulkkinen, T. I., Janhunen, P., and Wu, C.-C.: Stormtime energy transfer in global MHD simulation, J. Geophys. Res., 108(A1), 1048, doi:10.1029/2002JA009446, 2003.

Palmroth, M., Laitinen, T. V., and Pulkkinen, T. I.: Magnetopause energy and mass transfer: results from a global MHD simulation, Ann. Geophys., 24, 3467-3480, doi:10.5194/angeo-243467-2006, 2006.

Palmroth, M., Koskinen, H. E. J., Pulkkinen, T. I., Toivanen, P. K., Janhunen, P., Milan, S. E., and Lester, M., Magnetospheric feedback in solar wind energy transfer, J. Geophys. Res., 115, A00I10, doi:10.1029/2010JA015746, 2010.

Palmroth, M., Fear, R., and Laitinen, T. V.: Magnetopause energy transfer dependence on dipole tilt and solar wind parameters: A view from a global MHD simulation, in preparation, 2011.

Papadopoulos, K., Goodrich, C., Wiltberger, M., Lopez, R., and Lyon, J. G.: The physics of substorms as revealed by the ISTP, Phys. Chem. Earth, 24, 189-202, 1999.

Raeder, J.: Global Magnetohydrodynamics - A Tutorial, in: Space Plasma Simulation, edited by: Buechner, J., Dum, C. T., and Scholer, M., Lecture Notes in Physics, vol. 615, Springer Verlag, Heidelberg, 2003.

Rème, H., Aoustin, C., Bosqued, J. M., Dandouras, I., Lavraud, B., Sauvaud, J. A., Barthe, A., Bouyssou, J., Camus, Th., CoeurJoly, O., Cros, A., Cuvilo, J., Ducay, F., Garbarowitz, Y., Medale, J. L., Penou, E., Perrier, H., Romefort, D., Rouzaud, J., Vallat, C., Alcaydé, D., Jacquey, C., Mazelle, C., d'Uston, C., Möbius, E., Kistler, L. M., Crocker, K., Granoff, M., Mouikis, C., Popecki, M., Vosbury, M., Klecker, B., Hovestadt, D., Kucharek, H., Kuenneth, E., Paschmann, G., Scholer, M., Sckopke, N., Seidenschwang, E., Carlson, C. W., Curtis, D. W., Ingraham, C., Lin, R. P., McFadden, J. P., Parks, G. K., Phan, T., Formisano, V., Amata, E., Bavassano-Cattaneo, M. B., Baldetti, P., Bruno, R., Chionchio, G., Di Lellis, A., Marcucci, M. F., Pallocchia, G., Korth, A., Daly, P. W., Graeve, B., Rosenbauer, H., Vasyliunas, V., McCarthy, M., Wilber, M., Eliasson, L., Lundin, R., Olsen, S., Shelley, E. G., Fuselier, S., Ghielmetti, A. G., Lennartsson, W., Escoubet, C. P., Balsiger, H., Friedel, R., Cao, J.-B., Kovrazhkin, R. A., Papamastorakis, I., Pellat, R., Scudder, J., and Sonnerup, B.: First multispacecraft ion measurements in and near the Earth's magnetosphere with the identical Cluster ion spectrometry (CIS) experiment, Ann. Geophys., 19, 1303-1354, doi:10.5194/angeo19-1303-2001, 2001.

Rosenqvist, L., Buchert, S., Opgenoorth, H., Vaivads, A., and Lu, G.: Magnetospheric energy budget during huge geomagnetic activity using Cluster and ground-based data, J. Geophys. Res., 111, A10211, doi:10.1029/2006JA011608, 2006.

Rosenqvist, L., Opgenoorth, H. J., Rastaetter, L., Vaivads, A., and Dandouras, I.: Comparison of local energy conversion estimates from Cluster with global MHD simulations, Geophys. Res., Lett., 35, L21104, doi:10.1029/2008GL035854, 2008a.

Rosenqvist, L., Vaivads, A., Retinò, A., Phan, T., Opgenoorth, H. J., Dandouras, I., and Buchert, S.: Modulated reconnection rate and energy conversion at the magnetopause under steady IMF conditions, Geophys. Res., Lett., 35, L08104, doi:10.1029/2007GL032868, 2008b.

Shue, J.-H., Chao, J. K., Fu, H. C., Russell, C. T., Song, P., Khurana, K. K., and Singer, H. J.: A new functional form to study the solar wind control of the magnetopause size and shape, J. Geophys. 
Res., 102(A5), 9497-9511, doi:10.1029/97JA00196, 1997.

Shue, J.-H., Song, P., Russell, C. T., Steinberg, J. T., Chao, J. K., Zastenker, G., Vaisberg, O. L., Kokubun, S., Singer, H. J., Detman, T. R., and Kawano, H.: Magnetopause location under extreme solar wind conditions, J. Geophys. Res., 103, 1769117700, 1998.

Shukhtina, M. A., Gordeev, E. I., and Sergeev, V. A.: Timevarying magnetotail magnetic flux calculation: a test of the method, Ann. Geophys., 27, 1583-1591, doi:10.5194/angeo-271583-2009, 2009.

Siscoe, G. L. and Cummings, W. D.: On the cause of geomagnetic bays, Planet. Space Sci., 17, 1795-1802, 1969.

Smith, C. W., Acuna, M. H., Burlaga, L. F., Heureux, J. L., Ness, N. F., and Scheifele, J.: The ACE magnetic fields experiment, Space Sci. Rev., 86, 613-632, 1998.
Sonnerup, B. U. Ö., Haaland, S., Paschmann, G., Dunlop, M. W., Rème, H., and Balogh, A.: Orientation and motion of a plasma discontinuity from single-spacecraft measurements: Generic residue analysis of Cluster data, J. Geophys. Res., 111, A05203, doi:10.1029/2005JA011538, 2006.

Trenchi, L., Marcucci, M. F., Pallocchia, G., Consolini, G., Bavassano Cattaneo, M. B., Di Lellis, A. M., Rème, H., Kistler, L., Carr, C. M., and Cao, J. B., Occurrence of reconnection jets at the dayside magnetopause: Double Star observations, J. Geophys. Res., 113, A07S10, doi:10.1029/2007JA012774, 2008. 